\title{
La prise de décision éthique, notamment au vu de la constante limitation des ressources
}

\section{Jean Martina,}

Simone Romagnoli ${ }^{b}$

a Dr, Médecin de santé publique, membre de la CNE jusqu'à fin 2013

b $\mathrm{PhD}$, philosophe et éthicien, collaborateur scientifique de la CNE
La Commission nationale d'éthique (CNE) a été invitée à s'exprimer lors du $10^{\mathrm{e}}$ anniversaire de la Commissione di etica clinica (COMEC) du canton du Tessin, lors d'un symposium organisé à Bellinzone les 12 et 13 septembre 2013. La version italienne de l'exposé fait à cette occasion a été publiée par la «Rivista per le Medical Humanities» (rMH), sous le titre «Di fronte alla limitazione delle risorse» [1]. Le présent article, publié avec l'aimable accord de la rMH, est une version adaptée reprenant les éléments principaux de la présentation initiale.

\section{La Commission nationale d'éthique pour la médecine humaine}

\section{Composition}

La CNE [2] compte actuellement 15 membres parmi lesquels des professionnels de plusieurs disciplines: philosophie/théologie; santé et sciences biologiques; suggérés par tout un chacun et qui lui paraissent pertinents.

\section{Manière de travailler}

La CNE est un groupe d'experts, le plus souvent de niveau académique; ses membres auront donc l'habitude de débattre sur des sujets complexes. Les règles principales comprennent l'écoute attentive, le respect de l'autre et la recherche constructive d'un consensus grâce à une démarche délibérative (c'està-dire soumise à la réflexion critique et à l'argumentation rationnelle). Un fait de la vie auquel les membres du comité seront confrontés (que la plupart connaîtront d'expérience) est qu'il est fréquent que des problèmes n'admettent aucune solution optimale, mais seulement des mauvaises et des moins mauvaises. Il convient avec persévérance de rechercher la moins mauvaise des options. Un autre fait consiste à reconnaître l'existence de désaccords

\section{«Elle informe sur des thèmes importants et actuels dans le domaine bioéthique et encourage le dialogue public.»}

droit et autres domaines comme sciences sociales, représentation des intérêts des patients, économie. Langues et genres doivent y être équitablement représentés, ainsi que des courants éthiques et culturels divers.

\section{Mission}

Selon l'article 28 de la loi fédérale sur la procréation médicalement assistée, la CNE suit l'évolution dans les domaines des techniques de procréation et du génie génétique et donne des avis consultatifs sur les questions sociales, scientifiques et juridiques qui en résultent. L'ordonnance sur la CNE élargit les tâches qui lui sont dévolues, en précisant à l'article 1 qu'elle doit suivre «les développements scientifiques et leurs applications dans les domaines de la santé et de la maladie chez l'être humain» [3]. En ce sens, elle informe sur des thèmes importants et actuels dans le domaine bioéthique et encourage le dialogue public. Elle peut élaborer des directives; elle conseille, à la demande, le Parlement, le Conseil fédéral et les cantons. La Commission peut se saisir elle-même de certains thèmes, $y$ compris de ceux qui seraient moraux (d'où la nécessité de la recherche constructive d'un consensus), qui parfois peuvent se révéler insurmontables.

Il est hautement souhaitable que la plupart des avis et recommandations de la CNE fassent l'objet d'une formulation à laquelle toute la commission, ou une large majorité, puisse se rallier. Point d'importance: si elle entend être écoutée des autorités et du public, la commission doit élaborer des conclusions aussi claires et précises que possible. Comme de toute manière lesdits avis ne sont que consultatifs, les instances qui pourraient leur donner force exécutoire seront moins enclines à le faire si ces recommandations sont vagues ou manquent de direction.

\section{Considérations de base}

Pour répondre à la problématique du titre de ce texte, il faut en relever les enjeux. Le questionnement de l'éthique peut se résumer dans la formule: «Comment faire pour bien faire?». Questionnement qui doit composer avec le fait que la réflexion est toujours «située», qu'elle doit tenir compte du contexte - dans ses di- 


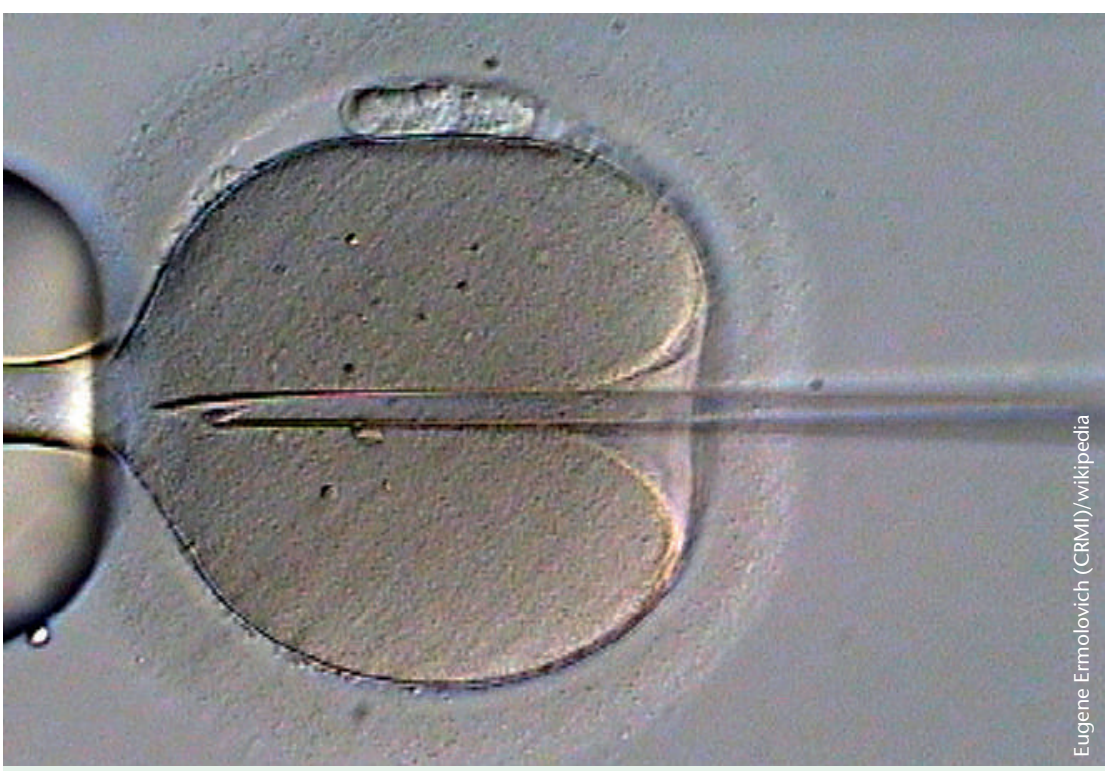

Entre autres la CNE suit l'évolution dans les domaines des techniques de procréation assistée.

verses dimensions - faute de quoi elle court le risque d'être dogmatique, non-pertinente et/ou inefficace.

\section{La prise de décision éthique}

Une prise de décision éthique est conditionnée par quatre éléments:

- la perspective de l'individu ou de l'institution qui l'effectue;

- le but poursuivi;

- la manière dont on procède (modalités de fonctionnement et de délibération; orientations éthiques utilisées);

- le contexte.

Rappelons qu'il y a en éthique plusieurs orientations possibles dont les deux suivantes: l'approche déontologique (certains principes doivent être appliqués indépendamment des résultats de cette application) et/vs. l'approche conséquentialiste (on se préoccupe des effets des orientations prises en cherchant à maximiser le bénéfice global qui peut être atteint).

En médecine individuelle et dans les soins, on suit l'adage «Salus aegroti suprema lex» (règle du médecin praticien-clinicien). Il est à concilier avec l'adage «Salus publica suprema lex», règle de l'action de santé publique (et idéalement de l'action politique - attention à la res publica). En pratique, il convient de chercher à concilier les approches individuelle et de santé publique et les différentes approches éthiques.

\section{La limitation des ressources}

On est toujours en situation de rareté de ressources, cela influence constamment la réflexion éthique:

- les limitations peuvent être momentanées ou durables, peuvent concerner les ressources humaines, matérielles ou en temps disponible;

- les limitations ou d'autres contraintes ont différentes origines (naturelles, humaines, morales, sociales, etc.) et engagent à ce titre de diverses manières la responsabilité des institutions et personnes concernées;

- les limitations peuvent ainsi être regrettables mais indépendantes de la responsabilité des acteurs ou partenaires/stakeholders (en termes familiers «c'est la faute à pas de chance», voire ce que les Anglo-Saxons appellent «acts of God»), ou injustes (c'est-à-dire le résultat de décisions discutables).

\section{Des niveaux différents}

Les limitations de ressources ont potentiellement des répercussions à plusieurs niveaux: micro-, mésoet macro-.

Au micro-niveau (relation thérapeutique), la réflexion éthique est d'abord celle du médecin et de l'équipe soignante, qui peut faire appel à un éthicien. Rappelons ici que, depuis 2013, si le patient n'a pas son discernement, n'a pas établi de directives anticipées et que sa situation requiert des décisions difficiles, c'est maintenant les proches (suivant une «cascade» fixée par le Code civil, l'article 378 notamment) qui ont la compétence de décider, dans la mesure où ils assurent une «assistance personnelle régulière» - et plus comme auparavant le médecin.

Au niveau méso- (structure de soins ambulatoires ou hôpital), les moyens d'assurer la qualité éthique des décisions devraient être mis en place par la direction (médicale, soignante et administrative), y compris par la création d'une commission d'éthique (clinique) de l'institution.

Au niveau macro- (entité socio-politique), le Gouvernement ou le Ministère de la santé propose au Parlement des orientations et des budgets. Une Commission d'éthique peut être sollicitée dans la préparation des programmes (idéalement, elle devrait l'être).

Si on entend apporter une vraie dimension scientifique, il est judicieux d'entreprendre des analyses multi-critères pour répondre à des questions comme: combien de ressources consacrées au diagnostic et au traitement, combien à la prévention; combien à des structures hospitalières, combien aux soins à domicile.

\section{Certaines orientations/décisions restent forcément du registre politique}

En ce qui concerne le macro-niveau, la liberté des parlementaires reste entière de voter des budgets qui facilitent ou au contraire rendent difficile la mise en place desdits programmes (NB: salus publica signifie aussi bien santé de la collectivité que salut/bien-être de celle-ci au sens plus général). 
Il faut également souligner que l'existence manifeste d'un besoin de santé n'entraîne pas automatiquement ou forcément que des ressources adéquates lui sont consacrées. «Par exemple, à financement donné [ressources limitées!], faut-il privilégier le soin des enfants ou celui des personnes âgées [cas de la démence par exemple]? Les humains n'ont donc pas d'autre alternative que de gérer leur vivre ensemble, leur cohabitation, dans la polis» [4]. Il importe de noter que, alors, on n'est que peu dans l'éthique et qu'on est pour l'essentiel dans la politique.

Dans notre système suisse, la pratique médicale libérale a certaines compétences et opportunités, l'Etat cantonal en a d'autres, l'Etat fédéral en a quelques-unes, les assureurs en ont aussi. Si l'un de ces stakeholders/partenaires est riche, il peut promouvoir dans son domaine de compétence des programmes qui potentiellement apporteront moins de bénéfices que si on faisait appel à un autre partenaire qui, en pratique, n'a pas assez de ressources pour agir. La répartition formelle des tâches entre différents niveaux et instances peut ainsi avoir des effets limitatifs du bénéfice de santé possible.

\section{Exemples tirés des travaux de la CNE}

Parmi la vingtaine de prises de position émises par la Commission nationale d'éthique depuis sa constitution en 2001, plusieurs sont clairement en rapport avec des enjeux éthiques liés à la limitation des ressources, notamment:

- Le soins médicaux: un devoir (No 8/2005);

- Plan suisse de pandémie influenza 2006 (et 2012, inchangé), dont le chapitre 10 , «Ethique», a été rédigé par la CNE. C'est là un exemple majeur du cas (hypothétique) où des choix engageant la survie de nombreuses personnes devraient être faits au vu de ressources et de temps disponible insuffisants.

- Les «déclarations éthiques de renonciation» mettent en péril le principe de solidarité de l'assurance-maladie (No 12/2006);

- Transplantation de foie d'une personne refusant toute transfusion de sang (cas des Témoins de Jéhovah) - Avis de juin 2008.

- Le consentement présumé en matière de don d'organes. Considérations éthiques (No 19/2012);
- Considérations éthiques sur le financement de l'avortement (No 21/2013);

\section{A propos de cette dernière prise de position}

Elle traite d'une initiative populaire qui entendait ne plus mettre à charge de l'assurance-maladie sociale (LAMal) les coûts liés à l'interruption de grossesse au sens du régime du délai accepté par le peuple suisse en 2002. Un argument des initiants était que les ressources mises à disposition des soins ne devraient pas être utilisées pour une procédure qu'ils considèrent comme dépendant du choix de la patiente. $\mathrm{La}$ CNE a relevé que, clairement, l'effet d'un tel refus de prise en charge serait de manière disproportionnée au détriment de personnes souvent fragilisées et de conditions socio-économiques précaires.

Point important: l'acceptation d'une telle mesure, au motif du «libre choix» de la personne, pourrait susciter des démarches qui toucheront d'autres membres de la collectivité: fumeurs, consommateurs d'alcool ou de drogues, personnes qui se comportent de manière téméraire (au volant ou en pratiquant des sports extrêmes), voire personnes en surpoids ou très sédentaires ne se maintenant pas en forme physique, personnes/familles qui refusent les vaccinations ou d'autres mesures préventives (et courent ainsi le risque de maladies évitables). Il y aurait là une véritable «pente glissante» qui renverserait le principe de solidarité sur lequel est fondée la LAMal - en lui faisant suivre les principes de l'assurance privée (régie par une loi différente, celle sur le contrat d'assurance).

\section{Références}

1 Rivista per le Medical Humanities (c/o Ente Ospedaliero Cantonale, Bellinzona). Vol. 8. numéro 27. Gennaio-Aprile 2014. p. 98-102.

2 Martin J. Le travail d'un comité national de bioéthique Questions de principe et de pratique. Bull Méd Suisses. 2009;90(11):438-41.

3 Ordonnance sur la Commission nationale d'éthique dans le domaine de la médecine humaine (OCNE) du 4 décembre 2000, RS 810.113, état le $1^{\text {er }}$ janvier 2012

4 Thiel MJ. Faites que je meure vivant - Vieillir, mourir, vivre. Paris: Bayard; 2013. 